\title{
Catheter-related blood stream infection caused by Raoultella ornithinolytica
}

\author{
Alicja Sękowska $^{1} \cdot$ Katarzyna Dylewska $^{2} \cdot$ Eugenia Gospodarek $^{1} \cdot$ Tomasz Bogiel $^{1}$
}

Received: 3 June 2014 / Accepted: 1 April 2015 / Published online: 19 April 2015

(C) The Author(s) 2015. This article is published with open access at Springerlink.com

\begin{abstract}
Raoultella spp. representatives are Gram-negative capsulated, nonmotile rods. These bacteria are found in the natural environment: plants, water, soil and insects. $R$. ornithinolytica is one of the three species of Raoultella. $R$. ornithinolytica is the only species within the genus which has the ability to produce ornithine decarboxylase. Human infections related to $R$. ornithinolytica are exceedingly rare. The present case report describes catheter-related blood stream infection caused by $R$. ornithinolytica and successfully treated with antibiotic therapy.
\end{abstract}

\section{Introduction}

Raoultella species are Gram-negative nonmotile rods belonging to Enterobacteriaceae family. Initially, Raoultella spp. were classified as Klebsiella spp., but on the basis of $16 \mathrm{~S}$ ribosomal RNA and $r p o B$ gene sequences, these two genera were discriminated (Drancourt et al. 2001). These bacteria are found in the natural environment and in animals. Human infections related to Raoultella spp. are exceedingly rare. Raoultella ornithinolytica was initially described as Klebsiella ornithinolytica by Sakazaki et al. in 1989 (Sakazaki et al. 1989). The first human infection caused by $R$. ornithinolytica was reported in 2009 (Morais et al.

Alicja Sękowska

asekowska@cm.umk.pl

1 Department of Microbiology, Nicolaus Copernicus University in Torun, Ludwik Rydygier Collegium Medicum in Bydgoszcz, 9 M. Skłodowskiej-Curie Street, 85-094 Bydgoszcz, Poland

2 Department of Paediatric, Hematology and Oncology, Nicolaus Copernicus University in Toruń, Ludwik Rydygier Collegium Medicum in Bydgoszcz, 9 M. Skłodowskiej-Curie Street, 85-094 Bydgoszcz, Poland
2009; Vos and Laureys 2009). However, the correct identification of Raoultella species is very difficult. By the conventional methods used routinely in the microbiological laboratory, Raoultella species are mostly indistinguishable from Klebsiella pneumoniae and Klebsiella oxytoca.

\section{Case report}

An 8-year-old child attended the Dr Antoni Jurasz University Hospital in Bydgoszcz with a fever. She had a history of brain tumour (retinoblastoma), a neurogenic bladder and frequent urinary tract infections. On admission to the paediatric clinic, the following laboratory tests were done: the peripheral blood counts: $\mathrm{WBC}-12.63 \times 10^{3} / \mu \mathrm{L}, \mathrm{RBC}-3.82 \times 10^{6} / \mu \mathrm{L}, \mathrm{HGB}-$ $11.0 \mathrm{~g} / \mathrm{dL}, \mathrm{MCV}-83.5 \mathrm{fL}$ and $\mathrm{HCT}-31.9 \%$. The procalcitonin level was $12 \mathrm{mg} / \mathrm{L}$, and C-reactive protein (CRP) level was $159 \mathrm{mg} / \mathrm{L}$. In view of the fever $\left(38.9^{\circ} \mathrm{C}\right)$, blood was taken routinely, as well as from a Broviac catheter, the latter both for microbiology tests and for blood culture. The tunnelled catheter was inserted 69 days before. Because of a neurogenic bladder, a urine sample was also collected and cultured.

After $8 \mathrm{~h}$ and $49 \mathrm{~min}$, a positive culture from the catheterderived blood was obtained; while after $11 \mathrm{~h}$ and $19 \mathrm{~min}$, a positive culture was also obtained. Both blood cultures yielded $R$. ornithinolytica. The strains demonstrated a mucoid colony on blood agar and mucoid pale pink colony on MacConkey agar. The strains were identified with an automated system: VITEK2 Compact (bioMérieux, biotype 4437711753066431, at a probability level of $87.0 \%$ ). The identification of the isolates was further confirmed using the technique called Matrix-Assisted Laser Desorption Ionization-Time of Flight Mass Spectrometry (MALDI-TOF MS) (Bruker, Germany). The assay was done twice to yield four scores: three for $R$. ornithinolytica scores-2.14-2.46-2.48 (medium 2.36) and 
one for $R$. planticola -2.02 . Thus, the obtained score $(\geq 2.300)$ indicated the correct identification at the species level.

$R$. ornithinolytica strains were sensitive to piperacillin, ticarcillin with clavulanic acid, piperacillin with tazobactam, cefuroxime, cefotaxime, ceftazidime, cefepime, imipenem, meropenem, amikacin, tobramycin, netilmicin, ciprofloxacin and cotrimoxazole. The strains were resistant to ampicillin and amoxicillin with clavulanic acid. The urine culture grew Proteus mirabilis sensitive to the following antibiotics: piperacillin, ticarcillin with clavulanic acid, piperacillin with tazobactam, cefuroxime, cefotaxime, ceftazidime, cefepime, imipenem, meropenem, amikacin, tobramycin, netilmicin, ciprofloxacin and cotrimoxazole. None of the cultured isolates produced extended-spectrum beta-lactamases. The child does not have any therapeutic agents at the time of acquiring the infection, neither has a rash.

The patient received courses of piperacillin with tazobactam $(4 \times 3.5 \mathrm{~g}$, i.v. $)$ and amikacin $(1 \times 500 \mathrm{mg}$, i.v. $)$ for 6 days. The procalcitonin level on the next day was $43 \mathrm{mg} / \mathrm{L}$ with CRP $261 \mathrm{mg} / \mathrm{L}$ and $192 \mathrm{mg} / \mathrm{L}$ on the third day. On the fourth day, both the blood culture and urine culture were negative; on the fifth day, CRP was $36 \mathrm{mg} / \mathrm{L}$. On the tenth day, both, the blood and urine samples, were taken for the follow-up control. The cultures were negative; the catheter was sterile, and the patient recovered. It suggests usefulness of the treatment applied.

\section{Discussion}

Rarely are Gram-negative rods isolated in catheter-related blood stream infections (CRBSI) (Nielsen et al. 2012). Catheter-related blood stream infections are usually caused by coagulase-positive staphylococci, especially Staphylococcus aureus, and coagulase-negative staphylococci. Raoultella spp. rods are primarily found in water, soil and plants. $R$. ornithinolytica was isolated from fish, ticks and termites.

Our case illustrates a CRBSI with a Gram-negative rod. Since $R$. ornithinolytica was first described in 2009 (Morais et al. 2009; Vos and Laureys 2009), only several cases of clinical infections in humans have been reported and none of catheter-related infection. Morais et al. identified the case of an enteric fever-like syndrome caused by $R$. ornithinolytica in an 82-year-old patient with a history of arterial hypertension and degenerative arthropathy. The patient was treated with amoxicillin with clavulanic acid. At the same time, Vos and Laureys reported colic distension associated with an inflammatory syndrome in a 97-year-old woman. The patient had a giant renal cyst. The woman was not treated with antibiotics, but the inflammatory syndrome resolved on its own. The third patient was an infant with visceral heterotaxy (Mau and Ross 2010). The child showed skin flushing, and the authors identified R. ornithinolytica. In 2011, Solak et al. (2011) identified $R$. ornithinolytica in a 44-year-old woman with diabetic foot lesion and a history of diabetes mellitus, hypertension, hypothyroidism and chronic kidney disease. The patient also had a rash on her legs. The patient was treated initially with piperacillin and tazobactam, and then with tigecycline, because of multidrug resistance. Some authors reported bacteraemia (Hadano et al. 2012) and urinary tract infections (García-Lozano et al. 2013) in cancer patients.

$R$. ornithinolytica are Gram-negative rods rarely found in human infections. Correct identification of these bacteria is still a challenge as they are possibly still being misidentified as Klebsiella on the basis of histamine production; many of the histamine-producing strains were identified as K. pneumoniae or $K$. oxytoca at first, but additional tests or methods later enabled their correct identification as Raoultella rods. Thus, $R$. planticola and $R$. ornithinolytica are histamine-producing bacteria as opposed to members of genus Klebsiella. The use of additional tests allows for the differentiation of these two bacterial genera. Rods of Raoultella genus can use histamine while Klebsiella ethanolamine as the only source of carbon in the medium. Moreover, Raoultella genus use 3-DL-betahydroxybutyric acid as the only source of carbon in the medium, but this property is also characteristic to selected Klebsiella strains. Species of $R$. planticola and $R$. ornithinolytica can grow at $4{ }^{\circ} \mathrm{C}$. $R$. ornithinolytica representatives produce ornithine decarboxylase and $R$. terrigena decompose of D-melezitose.

The MALDI-TOF MS technique is a rapid, simple and highthroughput proteomic system which appears to have been useful in bacteria identification (Van Veen et al. 2010). This is a soft ionization technique used in mass spectrometry, allowing the analysis of biomolecules (such as DNA, proteins and sugars) and large organic molecules (such as polymers and dendrimers), which tend to be fragile and fragmented when ionized by more conventional ionization methods. The MALDI-TOF MS technique demonstrated high sensitivity and specificity for $R$. ornithinolytica. The obtained score $(\geq 2.300)$ indicated the correct identification at the species level; while 2.000-2.299 at the genus level and probable at the species level, 1.700-1.999 indicated the probable identification at the genus level. This technique can be used for rapid identification of various microorganisms: aerobic and anaerobic bacteria, Gram-positive and Gram-negative bacteria as well as mycobacteria and yeasts.

Raoultella strains are usually sensitive to antibiotics (amoxicillin with clavulanic acid, piperacillin, piperacillin with tazobactam, cefuroxime, cefotaxime, ceftazidime, cefepime, imipenem, meropenem, ertapenem, gentamicin, amikacin, tobramycin, netilmicin, ciprofloxacin, levofloxacin, tigecycline and cotrimoxazole). However, Castanheira et al. (2009) described three strains of Raoultella spp. (two R. planticola and one $R$. ornithinolytica) isolated from patients; resistant to carbapenems, the drugs of choice when infections of multidrug resistant Gram-negative strains appear.

Our case report describes catheter-related bacteraemia caused by $R$. ornithinolytica, which was successfully treated 
with the applied antibiotic therapy (piperacillin with tazobactam and amikacin). As Raoultella spp. infections in humans are rare, the full implications of Raoultella as a human pathogen remain unknown.

Acknowledgments This research was financially supported by Nicolaus Copernicus University from funds for the maintenance of research in the Department of Microbiology.

Open Access This article is distributed under the terms of the Creative Commons Attribution 4.0 International License (http:// creativecommons.org/licenses/by/4.0/), which permits unrestricted use, distribution, and reproduction in any medium, provided you give appropriate credit to the original author(s) and the source, provide a link to the Creative Commons license, and indicate if changes were made.

\section{References}

Castanheira M, Deshpande LM, DiPersio JR, Kang J, Weinstein MP, Jones RN (2009) First descriptions of bla KPC in Raoultella spp. ( $R$. planticola and $R$. ornithinolytica): report from the SENTRY Antimicrobial Surveillance Program. J Clin Microbiol 47:4129-4130

Drancourt M, Bollet C, Carta A, Rousselier P (2001) Phylogenetic analyses of Klebsiella species delineate Klebsiella and Raoultella gen. nov., with description of Raoultella ornithinolytica comb. nov., Raoultella terrigena comb. nov. and Raoultella planticola comb. nov. Int J Syst Evol Microbiol 51:925-932
García-Lozano T, Pascual Plá FJ, Aznar Oroval E (2013) Raoultella ornithinolytica in urinary tract infections. Clinical and microbiological study of a series of 4 oncologic patients. Med Clin (Barc) 141:138 139. doi:10.1016/j.medcli.2012.11.021

Hadano Y, Tsukahara M, Ito K, Suzuki J, Kawamura I, Kurai H (2012) Raoultella ornithinolytica bacteremia in cancer patients: report of three cases. Intern Med 51:3193-3195

Mau N, Ross LA (2010) Raoultella ornithinolytica bacteremia in an infant with visceral heterotaxy. Pediatr Infect Dis J 29:1-2

Morais VP, Daporta MT, Bao AF, Campello MG, Andrés GQ (2009) Enteric fever-like syndrome caused by Raoultela ornithinolytica (Klebsiella ornithinolytica). J Clin Microbiol 47:868-869

Nielsen XC, Chen M, Blok Hellesøe AM, Bekker Jeppesen P, Gyldenlykke J, Tvede M (2012) Etiology and epidemiology of catheter related bloodstream infections in patients receiving home parenteral nutrition in a gastromedical center at a tertiary hospital in Denmark. Open Microbiol J 6:98-101

Sakazaki R, Tamura K, Kosako Y, Yoshizaki E (1989) Klebsiella ornithinolytica sp. nov., formerly known as ornithine-positive Klebsiella oxytoca. Curr Microbiol 18:201-206

Solak Y, Gul EE, Atalay H, Genc N, Tonbul HZ (2011) A rare human infection Raoultella ornithinolytica in a diabetic foot lesion. Ann Saudi Med 31:93-94

Van Veen SQ, Claas EC, Kuijper EJ (2010) High-throughput identification of bacteria and yeast by matrix-assisted laser desorption ionization-time of flight mass spectrometry in conventional medical microbiology laboratories. J Clin Microbiol 48:900-907

Vos B, Laureys M (2009) Giant renal cyst as cause of colic obstruction. Rev Med Brux 30:107-109 